\title{
Análisis de redes sociales en los estudiantes de la carrera de psicología clínica de la Universidad Central del Ecuador
}

Fecha de recepción : 10 de septiembre de 2019 • Fecha de aceptación: 8 de noviembre de 2019 • Fecha de publicación: 13 de enero de 2020

Patricia del Consuelo Lucero Albán

Universidad Central del Ecuador

patypsdayanara@hotmail.com

https://orcid.org/0000-0002-5448-6160

Luis Gonzalo Landeta Bejarano

Universidad Central del Ecuador luislandeta2000@hotmail.com

https://orcid.org/0000-0002-2985-3395

Teresa Loma Vaca

Universidad Central del Ecuador teresa.loma@yahoo.es https://orcid.org/0000-0002-8103-9550

Maria Soledad Vargas Gomez

Universidad Central del Ecuador

vsolmary@yahoo.es https://orcid.org/0000-0001-9867-0667

\section{Resumen}

El objetivo del presente estudio es determinar cuál es la red social que tiene mayor influencia en la vida estudiantil de los jóvenes y otros aspectos relevantes. Para ello en el trabajo se analiza una muestra aleatoria simple de 237 participantes y un documento estructurado: "Cuestionario de uso de las Redes Sociales". El mismo fue aplicado de manera presencial y fue diseñado con la finalidad de recopilar información proporcionada por los estudiantes; presenta doce cuestionamientos con forma- 
to de respuestas de tipo Likert. Los resultados dan a conocer que el $92 \%$ utiliza Facebook, el $92 \%$ lo utiliza para el cumplimiento de las actividades de tipo académico; llegando de esta forma a la conclusión de que para los jóvenes encuestados de entre las redes sociales Facebook es el principal medio de comunicación, los estudiantes utilizan las redes con fines académicos y de interrelacionarse con sus pares.

Palabras clave: Psicología, Redes sociales, comunicación, recursos.

\begin{abstract}
The objective of this study is to determine what is the social network that has the greatest influence on the student life of young people and other relevant aspects. For this, the paper analyzes a simple random sample of 237 participants and a structured document: "Questionnaire on the use of Social Networks". It was applied in person and was designed with the proposal to collect information specifically by students; It presents twelve questions with a Likert response format. The results show that $92 \%$ use Facebook, 92\% use it for the fulfillment of academic activities; coming to the conclusion that for the young respondents of social networks Facebook is the main means of communication, students use the networks for academic purposes and to interact with their peers.
\end{abstract}

Keywords: Psychology, Social networks, communication, resources. 


\section{Introducción}

El comportamiento de las personas jóvenes está sujeto a diversos factores entre los que se pueden mencionar a los personales en los que influye en gran parte la imagen de sí mismo, el estado físico, las etapas del ciclo de la vida que se caracterizan por conductas propias de cada una de ellas, el estilo de vida que obedece a patrones de interacción con el ambiente, la personalidad y el auto concepto, los factores sociales como el aspecto religioso, lo económico, político, y la familia.

Sin duda alguna los factores culturales que son los que han contribuido a que las sociedades hayan dado rienda suelta al desarrollo de tecnologías cada vez más avanzadas para mejorar la información y, por ende, la comunicación que cada día es màs veloz y eficiente.

Para nadie es desconocida la influencia que estos factores tienen en el comportamiento de los seres humanos, tanto en la niñez como en la adolescencia y de manera específica en la etapa juvenil, ya que es bien conocido que entre sus aspectos positivos destacan el permitir la comunicación a gran escala y a un tiempo record, se mantienen informados de lo que pasa en el mundo, pero así mismo también se conoce que modifican los hábitos que ya estaban establecidos, acaparan la atención de los usuarios al punto de que se distraen frecuentemente mientras realizan tareas escolares o se interesan en contenidos que pueden ser considerados no tan beneficiosos.

Clarificar el objetivo de estudio que es el de conocer la relación existente entre los estudiantes de Psicología Clínica y las redes sociales ayuda a determinar que "La globalización que afecta la economía, las finanzas, la política y las sociedades civiles a nivel doméstico y regional" (Fukuyama, 2004), el mundo entero ha sucumbido ante el potencial desarrollo tecnológico y junto a ello, por supuesto para los adultos jóvenes en etapas de formación académica conjuntamente con su profesionalización casi de la mano va también el uso y abuso de las redes sociales que de una u otra forma han logrado generar un cambio radical en el estilo de la vida de las personas, tanto es así que actualmente se cree que el mundo no será el de antaño sin Facebook, WhatsApp, Twitter o Instagram.

Lo cierto es que al hacer referencia a lo mencionado no solo como otro tipo de comunicación, también cabe resaltar el papel trascendental que juega la formación de las representaciones mentales provenientes de los estímulos tanto visuales, como auditivos y táctiles que provienen del medio exterior para ser procesados, codificados, clasificados e interpretados en la corteza cerebral, específicamente en las áreas de asociación correspondientes a los lóbulos occipital, temporal y frontal, que juega un papel fundamental en la coordinación de la cognición y la emoción (Ardila, 2013) y que está íntimamente relacionado con los mecanismos del procesamiento de las emociones, funciones ejecutivas, memoria, atención, gnosias, lenguaje, praxias, habilidades viso espaciales y cognición social.

\section{Desarrollo}

A nivel mundial, "Las redes sociales cambiaron la forma en la que nos comunicamos. Ahora 
estamos todo el tiempo conectados, recibiendo y enviando información constantemente. Gracias a las redes sociales podemos acceder rápidamente a información que antes no estaba disponible y hasta seguir los acontecimientos en tiempo real" (Lagrere A., 2016). Esto ha permitido que con el acceso a internet se lleve a la práctica la democratización del recurso comunicativo que ha producido grandes cambios en nuestra vida y su estilo.

Latinoamérica está considerada como la región que tiene mayor compromiso en cuanto al uso de redes sociales. Esta información hace referencia a que sus habitantes en promedio usan 6.0 horas al mes, por lo tanto es la región con el nivel más alto de visitantes en las redes sociales; frente a Asia Pacífico que presenta tan solo 2,2 horas y su nivel es el más bajo a nivel regional. También se informa que entre las "redes sociales más utilizadas en América Latina, aparecen primeros en la lista Facebook, Messenger y Linkedln, seguido por Twitter y Pinterest”. (comScore y Latamclick).

En el Ecuador, una publicación de metroecuador.com.ec (2014) indica que "el 17,11\% de la población mayor de 5 años, es decir 2,8 millones de ecuatorianos, declara usar redes sociales a través de su teléfono inteligente, mientras que según el Instituto Nacional de Estadísticas y Censos (INEC)" los mismos que al ser analizados con mayor detenimiento ponen en evidencia que esto puede ser a consecuencia del uso de los teléfonos inteligentes más conocidos como "Smartphones". Así los datos de que en el 2015 en Ecuador alrededor de 3'084.886 habitantes de distinta edad informaron tener uno de estos equipos, y en la población estudiantil universitaria se ha hecho muy evidente la influencia del uso de las redes sociales no solo en la conducta, ya que es muy notorio que aquellos que pertenecen a una determinada página o red social parecen mantener un mejor nivel de seguridad en sí mismo, más aún si gozan de cierto nivel de popularidad. En tanto, el rendimiento académico también puede verse afectado en la medida en que los estudiantes puedan tener acceso al internet y, por ende, a las páginas que aportan con amplia información digitalizada y que ofrecen una gran variedad de artículos con contenido actualizado.

Sin lugar a dudas con estos datos se evidencia que las personas parecen necesitar estar dentro de una red social sin que esto tenga que ver con su condición socioeconómica o nivel de instrucción académica. También el avance en lo que ha velocidad de conectividad se refiere ha ido en aumento en la última década, por ello hay muchos hogares que ya cuentan incluso con conexión de fibra óptica lo que les permite la transmisión de comunicaciones a gran velocidad y distancia. "La conectividad mejoró en el Ecuador y las personas estamos presentes en todas las redes sociales independientemente de la condición social, el acceso a internet es un tema que nos involucra a todos porque significa estar presente en el mundo de hoy", nos comparte Villafuerte (2016), ingeniero informático.

Así también cabe informar que en el Ecuador aproximadamente "4,9 millones de usuarios posee una cuenta en las redes sociales y pasan en ellas, en promedio 7,22 horas al día. El porcentaje de participación es mayor en el área urbana con el 81,9\% frente 18,1\% de participación del área rural". En cuanto al género tenemos otro dato interesante ya que son los varones los que más usan las redes sociales llegando a ser el $42 \%$ de la población encuestada quienes emplean 6,50 horas en promedio al día. Las personas consideradas dentro del grupo de mayores de edad con 
nivel de instrucción superior emplean hasta 7,26 horas diarias en las redes sociales (Encuesta de Condiciones de Vida, 2014). La forma actual en que fluye la comunicación ha convertido a las redes sociales en un nuevo fenómeno que ha logrado atrapar a las personas en su vida familiar, educativa y laboral hasta copar su tiempo libre.

Debido a ello, en el contexto universitario no deja de ser una alarmante preocupación para los docentes el uso y el abuso que los estudiantes dan a las distintas redes sociales, tanto en sus hogares como dentro de las aulas de estudio ,propiciando de esta forma el aparecimiento de nuevos comportamientos que pueden no ser bien aceptados en la comunidad académica como el acceso a redes sociales en horas clase y en otras actividades que no corresponden, para lo cual el presente estudio permitirá re direccionar la actuación del segmento de la población investigada con la intención de evitar que se generalicen ciertas conductas que tienen amplia repercusión en la calidad de vida de los sujetos investigados. La hipótesis planteada: ¿Es Facebook la red social más utilizada entre los estudiantes de la carrera de Psicología Clínica? Lo que permitirá evidenciar el impacto de la tecnología en la vida de un determinado grupo de estudiantes universitarios.

\section{Metodología}

Para la investigación se ha considerado un universo definido de 582 estudiantes en su totalidad, de la Carrera de Psicología Clínica de la Facultad de Ciencias Psicológicas en la Universidad Central del Ecuador, y una muestra aleatoria simple de 237 jóvenes de distintos cursos, con un margen error del $5 \%$.

Con los datos obtenidos se ha realizado un análisis estadístico a través de un cuestionario diseñado con 12 preguntas con respuestas tipo Likert ( $0=$ nuca, a veces $=1$, siempre $=2$ ), con una fiabilidad del $92 \%$, el mismo que generó resultados que serán de aporte para posteriores investigaciones relacionadas con la comunicación y el uso de las redes sociales de los estudiantes de la Carrera de Psicología Clínica de la Facultad de Ciencias Psicológicas, para lo cual se suman todos los valores de la escala y se asigna un valor de cero a 4 para un valor cualitativo de nunca, de 5 a16 su valor cualitativo de a veces y de 17 a 24 su valor cualitativo de siempre, la recolección de la información se inició el día 07 de junio del 2018 y finalizó el día 14 de julio del mismo año. Su aplicación obedeció al trámite del permiso respectivo por parte de la autoridad de turno, esto con el fin de proceder al ingreso de los investigadores a las aulas sorteadas, y una vez socializado el objetivo de la investigación procedieron a solicitar que lean el consentimiento informado; y finalmente les pidieron que respondan a la encuesta en físico.Los sujetos investigados tenían un promedio de edad de 22.8 años con una desviación estándar del 1.87 en más y en menos. El $74 \%$ corresponde al sexo femenino y el $26 \%$ al sexo masculino, y se encuentran cursando desde el primero al noveno semestre del periodo marzo 2018- agosto 2018.

\section{Resultados}

Se encontró que el $72 \%$ de la población investigada manifestó que siempre utiliza las redes sociales y a diario como un medio de comunicación el $28 \%$. El $92 \%$ de la población en estudio refiere que la red más utilizada es Facebook y el $8 \%$ considera otras redes sociales como el 
Instagram, que en este estudio está considerado como la red menos utilizada con un 55\%.

Tabla 1.

Aspectos Investigados

\begin{tabular}{llll}
\hline Aspectos Investigados & usan & no usan & Total \\
\hline Faacebook & $92 \%$ & $8 \%$ & $100 \%$ \\
Wathsaap & $72 \%$ & $28 \%$ & $100 \%$ \\
Telefonía Convencional & $15 \%$ & $85 \%$ & $100 \%$ \\
Tareas Académicas & $92 \%$ & $8 \%$ & $100 \%$ \\
Relaciones Afectivas & $72 \%$ & $28 \%$ & $100 \%$ \\
\hline
\end{tabular}

Elaborado por Luis Landeta

En la pregunta: ¿Considera que las imágenes que publica o comparte pueden generar conductas sexuales inadecuadas? el $68 \%$ responde que nunca, el $25 \%$ a veces y que siempre el $7 \%$.

¿Sueles revisar las redes sociales mientras estás en horas de comida?

Comentan que el $55 \%$ si revisa a veces y el $15 \%$ siempre revisa y un $30 \%$ que nunca lo realiza.

En la pregunta: ¿Permanece conectado a las redes sociales en las horas de sueño? Los estudiantes contestan que a veces el $47 \%$, mientras el $30 \%$, dice siempre y 2 por último el $3 \%$ responde que nunca.

\section{Discusión}

El 86\% de los internautas españoles comprendidos entre 16 y 65 años de edad se encuentran conectados a las redes sociales. Chatear el $67 \%$ actividades favoritas en las redes al igual que en el Ecuador, el $72 \%$ lo utilizan para comunicarse entre amistades, que son porcentajes similares en estas investigaciones.

El uso de las redes sociales está afectando los hábitos del sueño en los jóvenes mexicanos de los cuales el $70 \%$ se desvelan por estar conectados a estas aplicaciones (Instituto Mexicano de Medicina Integran del Sueño) al igual que en el Ecuador el 77\% manifiestan que se encuentran conectados a las redes sociales en las horas de sueño.

El Facebook en las universidades de los Estados Unidos se lo utiliza en un 98\% tanto por estudiantes como por docentes, cabe mencionar que este valor es superior al de la investigación que es solo el $92 \%$; estos valores seguirán cambiando según vayan usando otras herramientas informáticas.

Facebook es la red social usada por excelencia en España en un porcentaje del 91\%, de la misma manera en Ecuador se encuentra con el $92 \%$ que vienen a ser valores similares en estos países.

La Asociación Mexicana de la Industria Publicitaria y Comercial en Internet (2014) refiere que los 
hábitos de los usuarios en el internet sobre el uso diario son de 5 h 36 minutos.

Tomando en cuenta los resultados de la Revista de Educación a distancia (RED. Revista de Educación a distancia (http://www.um.es/ead/red/32) en un estudio realizado en un grupo de Universidades Argentinas, se llega a establecer que las Redes Sociales no tienen fines académicos, más bien son encaminados al cumplimiento de trámites administrativos, conocer el perfil de los usuarios y ampliar sus contactos entre los estudiantes, en tanto que en Ecuador el $92 \%$ de los investigados refieren que el Facebook cumple con una función académica debido posiblemente a que la página web que dispone la Universidad Central no cumple con todas las expectativas de los estudiantes, por ello es importante que se actualicen los servicios de esta página no solo desde el punto de vista de las necesidades de los docentes; sino también de los requerimientos estudiantiles como corresponde.

\section{Conclusiones}

- En la actualidad, el uso de las redes sociales se ha constituido en un medio indispensable de comunicación entre los estudiantes de la carrera de Psicología Clínica de la UCE. Los participantes en el proceso investigativo utilizan las redes sociales principalmente el Facebook para la comunicación entre compañeros y parejas, comparten principalmente la información de sus tareas académicas y la mayor parte de su tiempo están conectados a las redes sociales porque su prioridad es mantenerse actualizados con la información.

- El proceso investigativo denota que los estudiantes utilizan las redes con fines académicos y de interrelacionarse con sus pares; debiendo mencionar que la universidad debe potencializar el uso de la plataforma virtual UCE entre los miembros de la comunidad universitaria para que los estudiantes la utilicen también con fines académicos e investigativos.

- El uso de la tecnología va de la mano con la utilización de las redes sociales por lo que es innegable que los docentes universitarios deberían propiciar un entorno màs amigable que permita un mayor acercamiento entre estudiantes y profesores, lo que se verá reflejado en acciones vinculadas al desarrollo de actividades de trabajo autónomo u otras que se estimen para el efecto y que pretendan hacer uso de las redes sociales màs utilizadas por los estudiantes. 


\section{Referencias Bibliográficas}

Arab, E., Díaz, A. (2015). Impacto de las redes sociales e internet en la adolescencia: aspectos positivos y negativos. Revista Médica Clínica Las Condes, 26 (1).

Aria,R.(2014). LaAsociaciónMexicanadelaIndustriaPublicitariayComercialen Internet. SCRIP1(1)2-5. Recuperado https://es.scribd.com/document/247211144/Tecnologias-de-La-Informacion-y-La-Comunicacion-RUBY

Bocanelli, F. (2017). Estado de los medios sociales en América Latina 2016: datos de uso. Latamclick. Recuperado de https://www.latamclick.com/medios-sociales-en-america-latina-2016/

Brito, J. (2012). El uso de redes sociales por parte de las universidades a nivel institucional. Un estudio comparativo. Revista de Educación a distancia, 32(1). Recuperado de https://www.um.es/ead/red/32/laaser_et_al.pdf

Corbín, J. (s/a) Los 28 tipos de comunicación y sus características. Psicología y Mente. Recuperado https://psicologiaymente.net/social/tipos-comunicacion-caracteristicas.

Mendoza, H. Zambrano, K. Alcívar, E. (2015). Influencia de las redes sociales en la identidad personal de los universitarios chonenses. REVISTA CIENTIFICAS DOMINIO DE LAS CIENCIAS 1(1), 75-84.

Oliva, C. (2012). Redes sociales y jóvenes: una intimidad cuestionada en internet. Aposta Revista De Ciencias Sociales, 1 (54).

Tedesco, L. (2008). La era de la globalización: Estados bajo presión. Seminario en Madrid. Fundación para las Relaciones Internacionales y el Diálogo Exterior (3) 9-12 